\title{
Rolie-Poly fluid flowing through constrictions: Two distinct instabilities
}

\author{
T. Reis ${ }^{\mathrm{a}, *}$, H.J . Wilson ${ }^{\mathrm{b}}$ \\ ${ }^{a}$ OCCAM, Mathematical Institute, University of Oxford, Oxford, OX1 3LB, United \\ Kingdom \\ ${ }^{b}$ School of Mathematics, University College London, London, WC1E 6BT, United Kingdom
}

\begin{abstract}
Elastic instabilities of entangled polymer melts are common in industrial processes but the physics responsible is not well understood. We present a numerical linear stability study of a molecular based constitutive model which grants us physical insight into the underlying mechanics involved. Two constriction flows are considered - one shear dominated, the other extension dominated - and two distinct instabilities are found. The influence of the molecular structure and the behaviour of the polymer dynamics are investigated and in both cases chain relaxation and orientation play a crucial role. This suggests a molecular-based physical interpretation of the underlying mechanisms responsible for flow instabilities.
\end{abstract}

Keywords: Elastic instabilities; Rolie-Poly constitutive model; constriction flows

\section{Introduction}

Despite attracting much academic and industrial attention, a thorough and comprehensive understanding of the physics of bulk instabilities in viscoelastic flows remains elusive; if progress is to be made, one must consider the material's molecular properties. More precisely, knowledge of the connection between the underlying polymer dynamics and observed flow instabilities in linear polymeric melts is currently incomplete. This is due, in part, to the difficulties in modelling the molecular physics that result from a complicated underlying polymer architecture. The most adequate mathematical models for entangled polymer melts are, in general, extensions of the Doi-Edwards tube model [1] and should include the physical processes of reptation, convective constraint release (CCR), chain stretch and retraction. All these processes contribute to the rheology of the material and therefore it is natural to expect that each may also effect its stability properties. Moreover, since the rheological response is dependent on

*Corresponding author 
the dominant flow characteristics (for example, shear or elongational stretch), it is necessary to consider the influence of polymer dynamics in different flow geometries if the molecular physics of polymer instabilities is to be understood. Until recently, a mathematical model that was sufficiently sophisticated yet numerically tractable in inhomogeneous flow domains did not exist. In an attempt to further our understanding of the physics of elastic instabilities we present here an investigation of Rolie-Poly fluids [2] flowing through shear- and extensiondominated constrictions.

The first complete stability analysis of a Newtonian fluid was performed for two dimensional channel Poiseuille flow by Lin [3]. The onset of instability was found to occur at a Reynolds number of $R e=5776$. Non-Newtonian fluids, on the other-hand, have a complex microstructure and thus a complex rheological response which, in turn, effects the stability of the flow in a way considerably different to the Newtonian case. Ho and Denn [4] studied the stability properties of an upper convected Maxwell (UCM) fluid and found that the elasticity of the model had a stabilising effect at high Reynolds numbers. However, in contradiction to the numerical results, experimental data for low density polyethylene (LDPE) implies that elasticity has a destabilising effect. Wilson et al. [5] investigated the linear stability of UCM and Oldroyd-B fluids and Palmer and Phillips [6] extended this work to the PTT model, demonstrating the fluid to be linearly stable to infinitesimal disturbances in Poiseuille flow. Grillet et al. [7] considered the stability of the exponential PTT model and the Giesekus model using transient finite element calculations. The exponential PTT model was confirmed to be unstable in Couette and Poiseuille flow. The Giesekus model, on the other-hand, was shown to be unstable only in pressure-driven Poiseuille flow. The authors argue that the instabilities are caused by a coupling between the base state solution and perturbations to the velocity gradient. The stability boundaries of the Giesekus model have been numerically calculated by Öztekin et al. [8]. Like the PPT model, the extended Pom-Pom (XPP) has also been shown to be stable in both planar Couette and planar Poiseuille flow [9]. This is due to the XPP's non-zero second normal stress difference, which was shown to have a strong stabilising effect. Somasi and Khomami [10] numerically investigated the stability of viscoelastic fluids in plane Couette flow by simulating the Brownian dynamics of Hookean and FENE dumbbells, showing that stability is enhanced if the maximum extensibility of the dumbbells is decreased. Baltussen et al. [11] and Bogaerds et al. [12] numerically investigated viscoelastic instabilities in injection molding flows using the eXtended Pom-Pom (XPP) model. The authors show that this common industrial flow exhibits interfacial viscoelastic instabilities and find strain-hardening to have a stabilising effect. These results were verified by comparisons with experimental evidence. Theoretical studies of interfacial instabilities of viscoelastic materials can be found in $[13,14,15,16]$.

Theoretical and experimental studies have identified curved streamlines to be an influential mechanism responsible for instability in viscous and viscoelastic flows $[17,18,19]$. In particular, streamline curvature can couple with anisotropic stresses in the fluid, causing motion out of the plane of flow. Taylor-Couette flow is an example of a flow with curved streamlines that is known to become un- 
stable above some critical value. In Newtonian fluids, this instability is characterised by vorticity in the streamwise direction and the development of Goertler vortices: it is the inertial motion of material elements along curved streamlines that causes instability. Similar behaviour is observed with polymeric fluids and experiments show that viscoelastic materials demonstrate the existence of a purely elastic mode, i.e. an instability that occurs with negligible inertia $(R e \approx 0)$. If radial perturbations cause the polymer chains to be perturbed from a streamline then the chain becomes stretched by the shearing motion of the flow, which in turn amplifies the non-Newtonian hoop stress [19, 20]. By considering Taylor-Couette and lid-driven cavity flows, Pakdel and McKinley [19] developed a dimensionless quantity to characterise the critical conditions which determine the onset of elastic instabilities. Through geometric arguments and a suitable definition of the characteristic lengthscale of the relaxation of perturbations along a streamline, the relevant criterion was shown to be

$$
\left[\frac{l N_{1}}{R \sigma_{x y}}\right]^{\frac{1}{2}}>M=O(1),
$$

where $l=U \tau$ is the lengthscale along which the stress decays, $U$ is a characteristic velocity, $\tau$ is the reptation relaxation time, $N_{1}=\sigma_{x x}-\sigma_{y y}$ is the first normal stress difference, $\sigma_{x y}$ is the total shear stress, and $R$ is the radius of curvature of the streamline. This approach has recently been extended to find the criteria for instabilities in shear-banding fluids [21]. The dimensionless grouping defined by Pakdel and McKinley [19] given in equation (1) provides a general understanding of the curved streamline instability in elastic flows in terms of the relevant characteristic scales, but a detailed connection with the underlying physics remains elusive. In particular, although it is understood that a fluid's elasticity is crucial, to the best of our knowledge there has been little attempt to explain elastic instabilities in terms of the underling relaxation mechanisms - convective-constraint release, reptation and retraction - each of which contributes to the amount of stretching a polymer chain experiences in a given flow. Furthermore, it remains unclear whether the curved streamline mechanism is responsible for the instabilities that are observed in the somewhat more complicated constriction flows that are utilised in polymer processing.

An understanding of the physics behind viscoelastic flow instabilities is not only of pure scientific interest; it is also particularly relevant to the polymer processing industry since avoidance of instabilities could potentially save much wasted product and increase efficiency. The flow geometries that occur in industrial practice are generally complex and yield a rich display of rheological behaviour. Contraction-expansion flows, which contain elements of strong extension, encourage the non-Newtonian phenomena often observed in manufacturing. Futhermore, constriction flows of polymeric materials have recently been used to help determine the mechanisms that cause instabilities in the manufacturing of plastics. Collis et al. [22] compared numerical and experimental predictions for the complex flow behaviour of monodispersed polymer melts and argued that a very narrow polydispersity will amplify the flow in- 
stabilities that break lateral symmetry. However, no stability calculations were performed. Hassell et al. [23] present an experimental study of the formation of a polymer flow instability seen in a range of polystyrene melts flowing through contraction-expansion slit geometries and found that the underlying instability mechanism - an oscillating flow perpendicular to the bulk flow - is the same for both polydispersed and monodispersed materials. Although no single critical Weissenberg number was found, stability maps of Weissenberg numbers against material molecular weight in relation to both orientation and chain stretch were obtained. Su and Khomami [24] study instabilities in slit and converging channel die geometries using both asymptotic and numerical techniques. Their results suggest that material parameters (elasticity and viscosity), as well as the geometric design of the die, affect the interfacial instability. Hassell et al. [25] present stability measurements for polystyrene in a contraction-expansion geometry using both experimental and computational methods. The experimentally observed instability is confirmed using a numerical tool based on the Rolie-Poly constitutive equation [2] and the authors argue that chain stretch plays an important role. However, a detailed understanding of the relationship between polymer physics and engineering instabilities has still not been accomplished.

The numerical simulation of instabilities in viscoelastic flows has proved to be a major challenge in non-Newtonian fluid mechanics. Smith et al. [26] were arguably the first to present a robust numerical scheme that could accurately predict the onset of instability at the correct (experimentally verified) critical Weissenberg number for the flow of an Oldroyd-B fluid over a periodic array of confined cylinders. The only noticeable discrepancy between the numerical and experimental results is in the leading eigenfunction: the experiments show a periodic asymmetry [27] while the numerics predicted a steady symmetric state. Using a high resolution finite volume method in non-orthogonal block structured meshes, Alves, Pinho and Oliveira [28] also studied the flow of Maxwell and Oldroyd-B type fluids over confined cylinders and were able to obtain accurate predications for the drag coefficient. The method has yet to be extended to stability calculations. Poole et al. [29] numerically investigated polymer solutions flowing in sudden expansions using the PTT model and conclusively showed that the affect of elasticity is to reduce both the length and intensity of the recirculation region downstream of the expansion. More recently, Sahin and Wilson [30] presented an analysis of the stability of steady two dimensional Oldroyd-B flow over a periodic array of cylinders using a stylised finite volume method [31]. The implementation of the MUMPS library [32] together with the shift-invert Arnoldi method [33], as used by Sahin and Wilson [30], allows for efficient computations for very large eigenvalue systems on modern parallel architectures, and is utilsed here.

This article numerically investigates the polymer physics of flow instabilities. More precisely, the computational method first presented in [30] is applied to the Rolie-Poly constitutive model of Likhtman and Graham [2] to obtain a detailed understanding of the physical (molecular) mechanisms and critical parameters responsible for the onset of instabilities in the flow of monodispersed linear polymers. Since the rheological response of a fluid is dependent on the domi- 
nant flow characteristics, we study two flows through constrictions: the shear dominated periodic array of half-cylinders placed on channel walls (hereafter referred to the bumpy wall flow); and the extension dominated 8:1:8 contraction expansion slit geometry flow. Both domains are relevant to a general class of polymer-processing flows and demonstrates distinct instability mechanisms. The remainder of this article is organised as follows: in Section 2 we explain the equations of motion and the numerical procedure for hunting instabilities; in Section 3 we present our obtained results and identify the mechanism for instability in flows of monodispersed linear polymers; and concluding remarks are given in Section 4.

\section{Methodology}

The vast majority of previous work concerning the prediction of instabilities that occur in non-Newtonian flow has been limited to relatively conventional, well-known models, such as the UCM [4, 34, 35] and Oldroyd-B [26, 30] models. Such constitutive models for the polymeric contribution to the stress tensor exhibit many viscoelastic properties but their relative simplicity often renders them inadequate for realistic engineering applications. Moreover, it is not possible to obtain a comprehensive theoretical understanding of the mechanism for instabilities in viscoelastic flows without fully considering the underlying molecular physics. A powerful, kinetically-derived, constitutive equation for the extra stress is given by the Rolie-Poly model [2], currently the most advanced differential formulation of the Doi-Edwards tube model for linear polymer melts that is also compact enough to be computable in numerical calculations of spatially inhomogeneous flows. It incorporates the physics of reptation, convective constraint release (CCR), chain stretch, and retraction.

The Rolie-Poly constitutive equation for the extra-stress $\mathbf{T}$ may be written as

$$
\begin{aligned}
& \frac{\partial \mathbf{T}}{\partial t}+(\mathbf{u} \cdot \nabla) \mathbf{T}-(\nabla \mathbf{u}) \mathbf{T}-\mathbf{T}(\nabla \mathbf{u})^{\dagger} \\
& \quad=-\frac{1}{\tau}(\mathbf{T}-\mathbf{I})-\frac{2(1-\sqrt{3 / t r \mathbf{T}})}{\tau_{R}}\left(\mathbf{T}+\beta^{\star}\left(\frac{\operatorname{tr} \mathbf{T}}{3}\right)^{\delta} \mathbf{T}-\mathbf{I}\right)
\end{aligned}
$$

where $\tau$ is the reptation relaxation time, $\tau_{R}$ is the Rouse relaxation time, $\beta^{\star}$ is the convective-constraint release (CCR) parameter, $\mathbf{I}$ is the identity matrix, $\dagger$ denotes the matrix transpose, and $t r$ denotes the trace of a tensor. The parameter $\delta$ is taken to be $-1 / 2$ but may be fitted to experimental data [2]. The ratio of the two relaxation times defines the entanglement number, $Z$ [36]:

$$
Z=\frac{\tau}{3 \tau_{R}}
$$

We note that the Rolie-Poly module reduces to the Oldroyd-B model when $2(1-\sqrt{3 / \operatorname{tr} \mathbf{T}}) / \tau_{R} \rightarrow 0$. 


\subsection{Rheology of the Rolie-Poly model}

Before embarking on a stability analysis of the Rolie-Poly model in constriction flows, we first discuss its rheological properties in steady shear and planar extensional flow. This will help us understand the numerical results presented in Section 3 and to connect the stability mechanisms to the underlying polymer architecture.

We solve the Rolie-Poly equation (2) in simple shear flow for different entanglement ratios, $Z$, (equivalently, different relaxation-time ratios) and values of the CCR parameter, $\beta^{\star}$. Figures 1 shows the shear stress, $T_{x y}$, and the first normal stress difference, $N_{1}=T_{x x}-T_{y y}$, when $Z=5 / 3$ (that is, $\tau / \tau_{R}=5$ ). The case when $Z=20 / 3$ is shown in Figure 2 . We clearly see that the constitutive curve depends on both the relaxation-time ratio and the CCR parameter; the larger ratios resulting in smaller stresses. The Rolie-Poly model can predict non-monotonic behaviour, depending on the values of these quantities. Figure 2 shows that, for $3 Z=20$, monotonicity is lost for large shear-rates if $\beta^{\star} \lesssim 0.1$. This is a characteristic of steady shear-banding, which can be a source of interfacial instability [16]. The first normal stress difference, $N_{1}$ monotonically increases with shear rate in all cases.

We solve equation (2) in planar extensional flow for different relaxation-time ratios and values of the CCR parameter $\beta^{\star}$. Figure 3 shows the extensional viscosity, $\eta_{E}$, and the second normal stress difference, $N_{2}=T_{z z}-T_{y y}$, as a function of the strain rate, $\dot{\epsilon}$, when $Z=5 / 3$. The case when $Z=20 / 3$ is shown in Figure 4. After an initial plateau the fluid strain-softens before very rapidly hardening and diverging. Higher Weissenberg number ratios and more convective-constraint-release permit greater variation in the extensional viscosity, although the influence of CCR is considerably weaker. The second normal stress difference, $N_{2}$, undergoes a large variation in the range $0<\dot{\epsilon} \tau_{R}<$ 1 ; initially growing before a rapid decrease with increasing strain-rate, tending to its minimum value as the extensional viscosity diverges. It should be noted that, similar to the Oldroyd-B model, this singular behaviour occurs at $\dot{\epsilon} \tau_{R}=1$ $\left(\eta_{E} \rightarrow \infty\right.$ when $\left.\dot{\epsilon} \tau_{R} \rightarrow 1\right)$. We also notice the very steep gradient in the second normal stress difference for high strain-rates when $\beta^{\star}$ is large. The significance of these properties will be discussed further in Sections 3 and 4 . We note in passing that there exists a real solution as we approach the singularity from the right, although it is deemed unphysical.

\subsection{Non-dimensional governing equations}

The governing equations are the mathematical statements of the conservation of mass and momentum:

$$
\begin{aligned}
\nabla \cdot \mathbf{u} & =0 \\
\operatorname{Re}\left[\frac{\partial \mathbf{u}}{\partial t}+(\mathbf{u} \cdot \nabla) \mathbf{u}\right]+\nabla p & =\beta \nabla^{2} \mathbf{u}+\nabla \cdot \mathbf{T},
\end{aligned}
$$

where $R e$ is the Reynolds number, $\mathbf{u}$ is the velocity, $P$ is the pressure, and $\beta=\eta_{s} /\left(\eta_{s}+\eta_{p}\right)$ is the viscosity ratio of the solvent viscosity $\left(\eta_{s}\right)$ to the total 

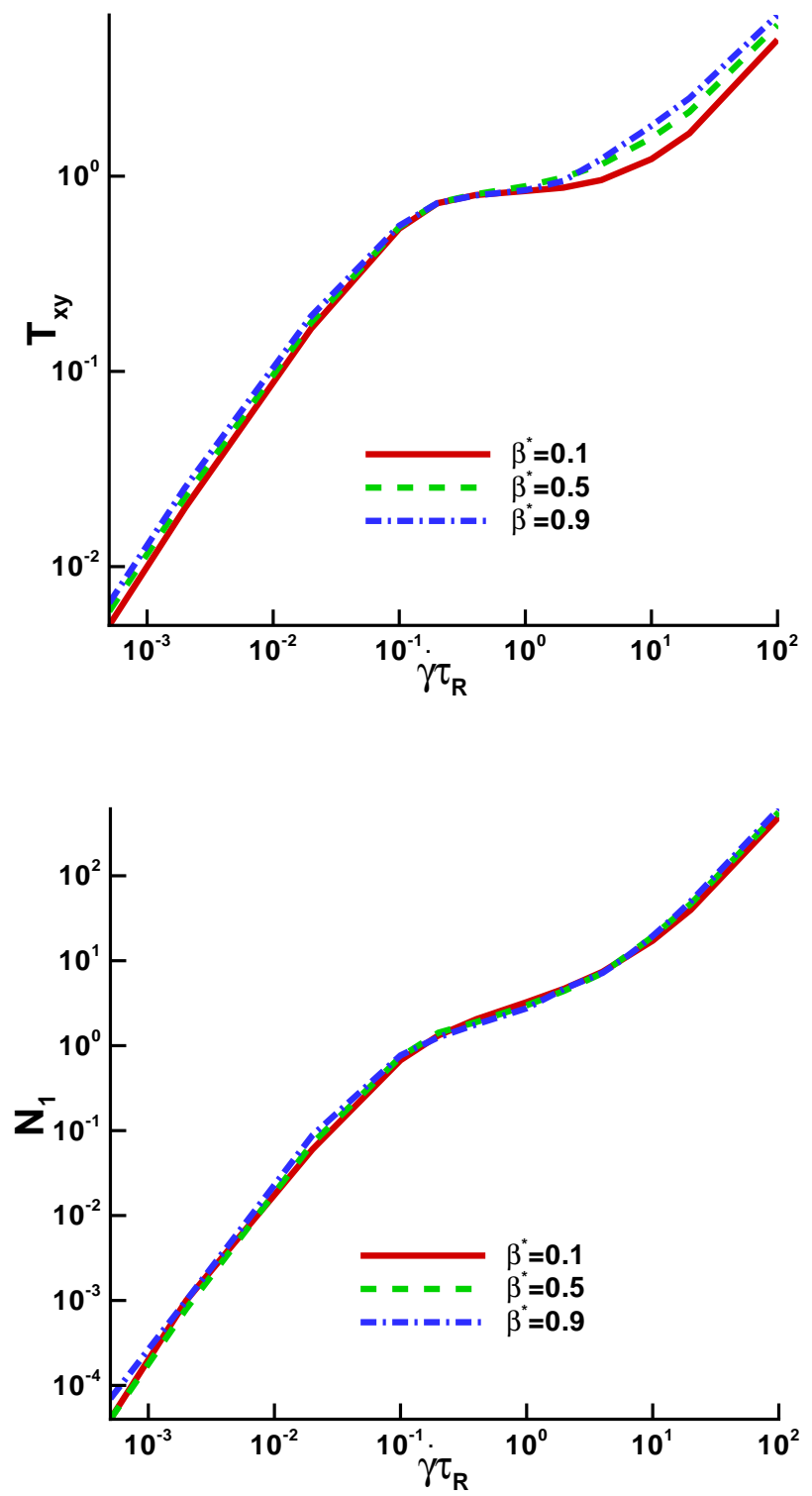

Figure 1: Plots of $T_{x y}$ and $N_{1}$ as a function of shear-rate when $3 Z=5$. 

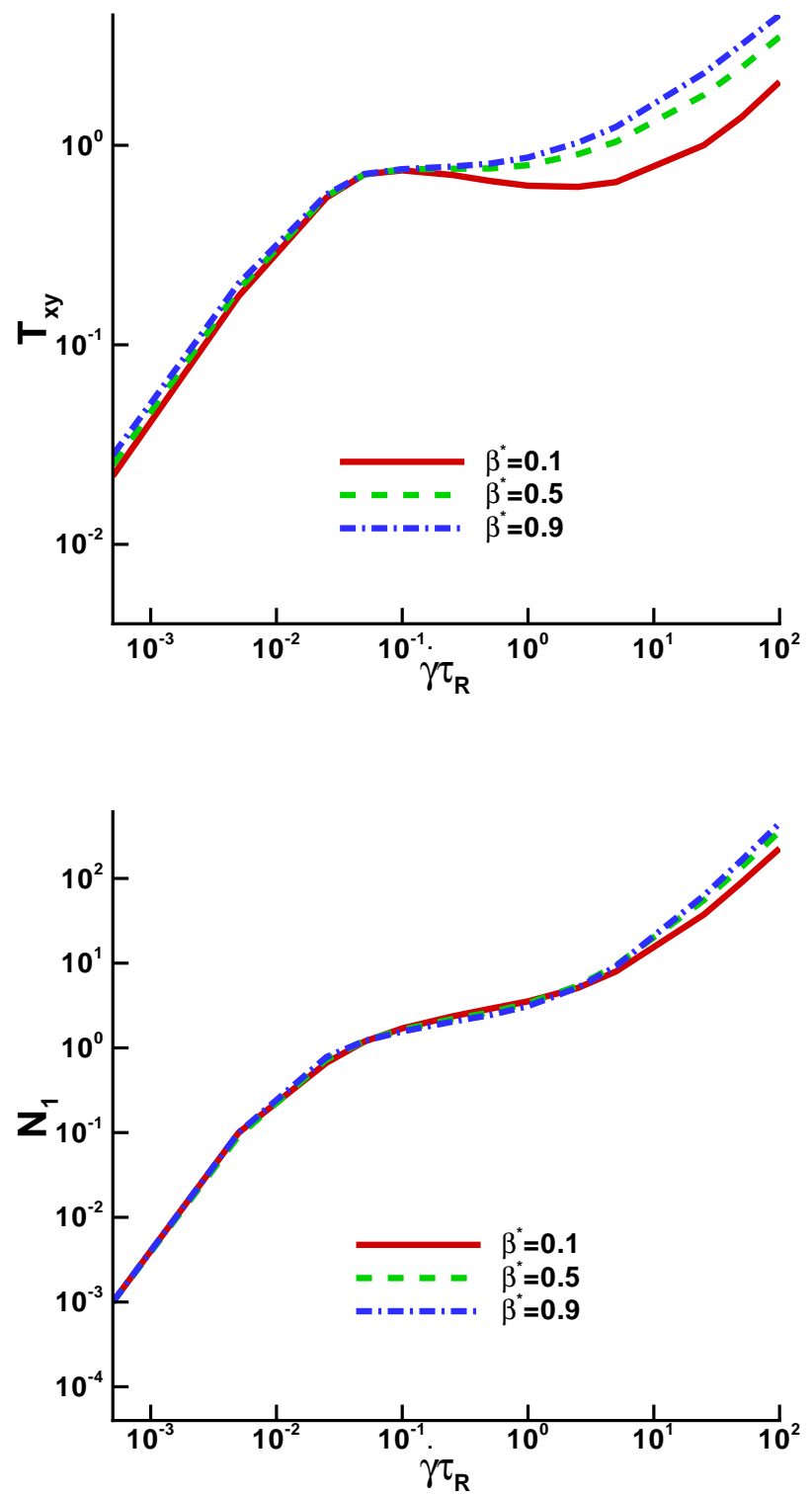

Figure 2: Plots of $T_{x y}$ and $N_{1}$ as a function of shear-rate when $3 Z=20$. 

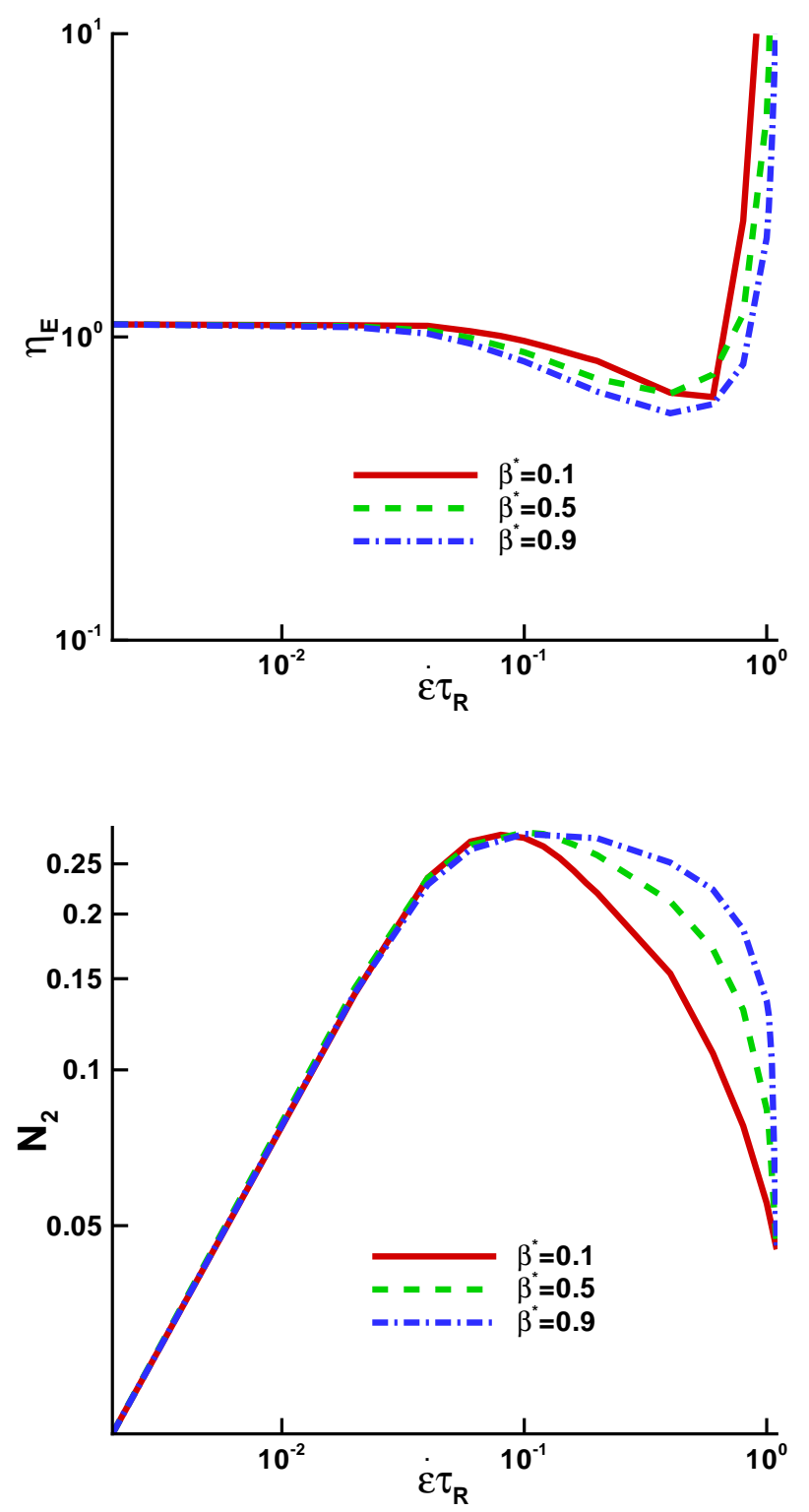

Figure 3: Plots of extensional viscosity, $\eta_{E}$, and $N_{2}$ as a function of strain-rate when $3 Z=5$. 

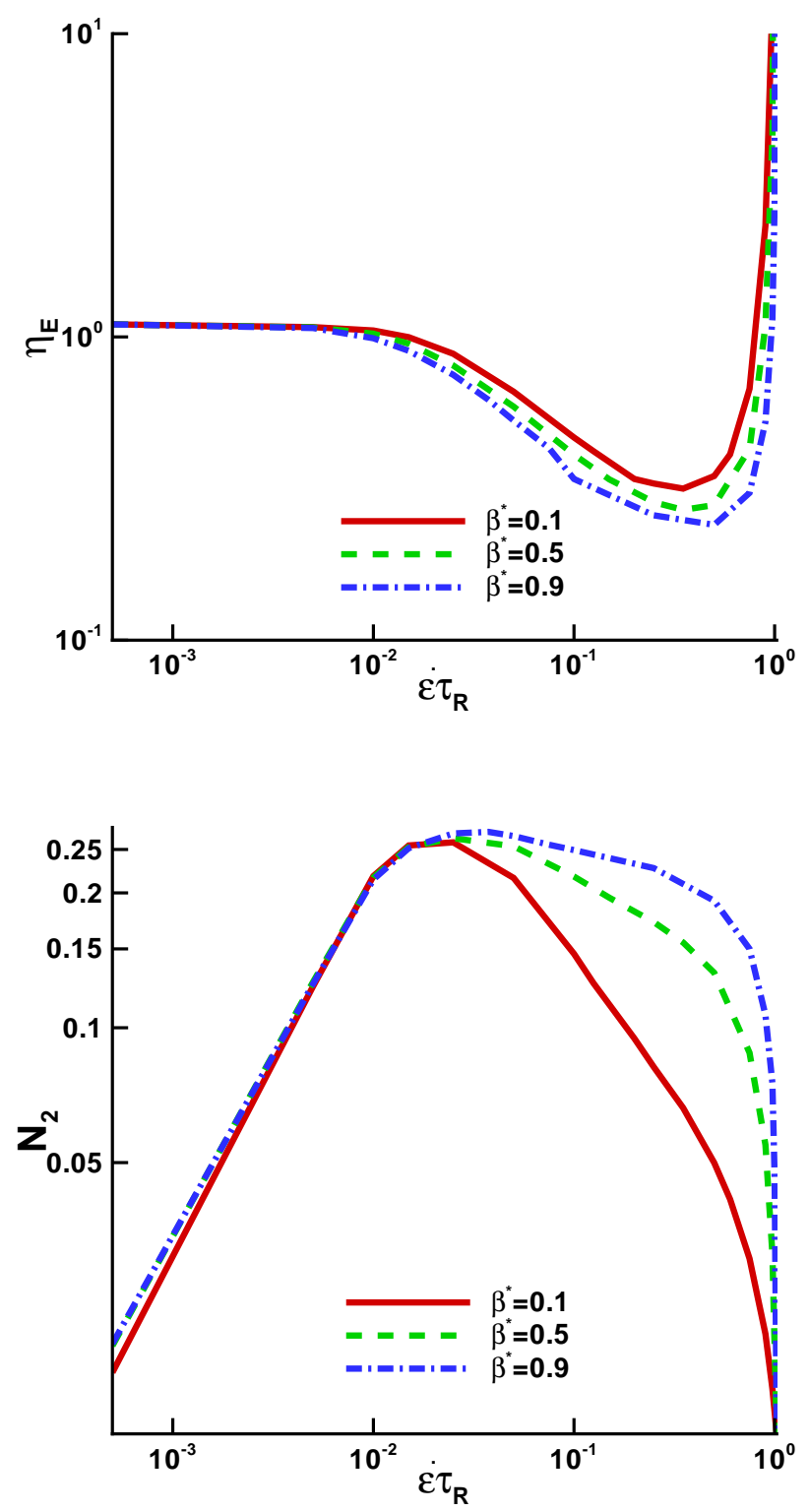

Figure 4: Plots of extensional viscosity, $\eta_{E}$, and $N_{2}$ as a function of strain-rate when $3 Z=20$. 
the viscosity $\left(\eta_{p}\right.$ being the polymer viscosity). We non-dimesnionalise the RoliePoly constitutive equation (2) for the extra stress:

$$
\begin{aligned}
W e & {\left[\frac{\partial \mathbf{T}}{\partial t}+(\mathbf{u} \cdot \nabla) \mathbf{T}-(\nabla \mathbf{u}) \mathbf{T}-\mathbf{T}(\nabla \mathbf{u})^{\dagger}\right] } \\
= & (1-\beta)\left(\nabla \mathbf{u}+(\nabla \mathbf{u})^{\dagger}\right)-\mathbf{T}\left(1+\frac{2 W e}{W e_{R}}\right)-\frac{2(1-\beta)}{W e_{R}} \mathbf{I}(1-\sqrt{3 / \sigma}) \\
& +\frac{2 W e \sqrt{3 / \sigma}}{W e_{R}} \mathbf{T}\left(1-\beta^{\star}\right)+\frac{6 W e \beta^{\star} / \sigma}{W e_{R}} \mathbf{T}
\end{aligned}
$$

where $W e$ and $W e_{R}$ are the Weissenberg numbers based on the reptation time $(\tau)$ and Rouse time $\left(\tau_{R}\right)$, respectively, and

$$
\sigma=\frac{W e}{1-\beta} \operatorname{tr} \mathbf{T}+\operatorname{tr} \mathbf{I}
$$

Equation (6) incorporates three relaxation mechanisms on each segment of the imagined polymer-constraining tube: the curvilinear diffusion of the chain along its center (reptation); the release of entanglement constraints due to the flow of the surrounding chains (CCR); and also the retraction of the chain back into the tube, which acts along the tube contour while holding the total length fixed at its equilibrium value. Convective constraint release is a more influential relaxation mechanism at higher shear rates.

\subsection{Perturbed Equations}

To analyse the linear stability of a system, theoretically or numerically, a base state which is independent of time is sought. This steady solution is then given a "kick"; that is, a small perturbation is added to the variables and decomposed into Fourier modes. The evolving system can be linearised about the base state with the resulting system having exponential behaviour in time. In other words, we consider three dimensional arbitrary infinitesimal disturbances to the two dimensional base flow, which can be written mathematically as follows:

$$
\mathbf{T}=\mathbf{T}_{B}+\hat{\mathbf{T}} e^{i k z+\omega t}, \quad \mathbf{u}=\mathbf{u}_{B}+\hat{\mathbf{u}} e^{i k z+\omega t}, \quad p=p_{B}+\hat{p} e^{i k z+\omega t},
$$

where $k$ is the wave number, $\omega$ is the growth rate and $i=\sqrt{-1}$. The subscripts $B$ denote the steady base flow. The components of $\hat{\mathbf{T}}, \hat{\mathbf{u}}$ and $\hat{p}$ are eigenvectors which are complex functions of $x$ and $y$ and have infinitesimal magnitude such that non linear terms can be neglected. The perturbed equations for mass and momentum are then

$$
\begin{aligned}
& \frac{\partial \hat{u}_{x}}{\partial x}+\frac{\partial \hat{u}_{y}}{\partial y}+k \hat{u} z=0, \\
& \operatorname{Re}\left[\omega \hat{u}_{x}+u_{B x} \frac{\partial \hat{u}_{x}}{\partial x}+u_{B y} \frac{\partial \hat{u}_{x}}{\partial y}+\hat{u}_{x} \frac{\partial u_{B x}}{\partial x}+\hat{u}_{y} \frac{\partial u_{B x}}{\partial y}\right]+\frac{\partial \hat{p}}{\partial x}
\end{aligned}
$$




$$
\begin{gathered}
=\beta\left[\frac{\partial^{2} \hat{u}_{x}}{\partial x^{2}}+\frac{\partial^{2} \hat{u}_{x}}{\partial y^{2}}-k^{2} \hat{u}_{x}\right]+\frac{\partial \hat{T}_{x x}}{\partial x}+\frac{\partial \hat{T}_{x y}}{\partial y}+k \hat{T}_{x x}, \\
\operatorname{Re}\left[\omega \hat{u}_{y}+u_{B y} \frac{\partial \hat{u}_{y}}{\partial x}+u_{B y} \frac{\partial \hat{u}_{y}}{\partial y}+\hat{u}_{x} \frac{\partial u_{B y}}{\partial x}+\hat{u}_{y} \frac{\partial u_{B y}}{\partial y}\right]+\frac{\partial \hat{p}}{\partial y} \\
=\beta\left[\frac{\partial^{2} \hat{u}_{y}}{\partial x^{2}}+\frac{\partial^{2} \hat{u}_{y}}{\partial y^{2}}-k^{2} \hat{u}_{y}\right]+\frac{\partial \hat{T}_{x y}}{\partial x}+\frac{\partial \hat{T}_{y y}}{\partial y}+k \hat{T}_{y x}, \\
\operatorname{Re}\left[\omega \hat{u}_{z}+u_{B x} \frac{\partial \hat{u}_{z}}{\partial x}+u_{B y} \frac{\partial \hat{u}_{z}}{\partial y}\right]-k \hat{p} \\
=\beta\left[\frac{\partial^{2} \hat{u}_{z}}{\partial x^{2}}+\frac{\partial^{2} \hat{u}_{z}}{\partial y^{2}}-k^{2} \hat{u}_{z}\right]+\frac{\partial \hat{T}_{x z}}{\partial x}+\frac{\partial \hat{T}_{y z}}{\partial y}+k \hat{T}_{z z},
\end{gathered}
$$

while those for the Rolie-Poly constitutive equation are

$$
\begin{aligned}
& W e\left[\omega \hat{T}_{x x}+u_{B x} \frac{\partial \hat{T}_{x x}}{\partial x}+u_{B y} \frac{\partial \hat{T}_{x x}}{\partial y}+\hat{u}_{x} \frac{\partial T_{B x x}}{\partial x}+\hat{u}_{y} \frac{\partial T_{B x x}}{\partial y}\right]- \\
& 2 W e\left[\frac{\partial \hat{u}_{x}}{\partial x} T_{B x x}+\frac{\partial \hat{u}_{x}}{\partial y} T_{B x y}+\frac{\partial u_{B x}}{\partial x} \hat{T}_{x x}+\frac{\partial u_{B x}}{\partial y} \hat{T}_{x y}\right] \\
& =2(1-\beta) \frac{\partial \hat{u}_{x}}{\partial x}-2 \frac{W e}{W e_{R}} \hat{T}_{x x}\left(\frac{\sigma+3 \beta^{\star}}{\sigma}-\frac{\sqrt{3}\left(1-\beta^{\star}\right)}{\sqrt{\sigma}}\right) \\
& -\frac{T_{B x x} W e^{2} \operatorname{tr} \hat{\mathbf{T}}}{\sigma W e_{R}(1-\beta)}\left(\frac{\sqrt{3}\left(1-\beta^{\star}\right)}{\sqrt{\sigma}}+\frac{6 \beta^{\star}}{\sigma}\right)-\frac{W e \sqrt{3 / \sigma} t r \hat{\mathbf{T}}}{W e_{R} \sigma}-\hat{T}_{x x}, \\
& W e\left[\omega \hat{T}_{y y}+u_{B x} \frac{\partial \hat{T}_{y y}}{\partial x}+u_{B y} \frac{\partial \hat{T}_{y y}}{\partial y}+\hat{u}_{x} \frac{\partial T_{B y y}}{\partial x}+\hat{u}_{y} \frac{\partial T_{B y y}}{\partial y}\right]- \\
& 2 W e\left[\frac{\partial \hat{u}_{y}}{\partial x} T_{B x y}+\frac{\partial \hat{u}_{y}}{\partial y} T_{B y y}+\frac{\partial u_{B y}}{\partial x} \hat{T}_{x y}+\frac{\partial u_{B y}}{\partial y} \hat{T}_{y y}\right] \\
& =2(1-\beta) \frac{\partial \hat{u}_{y}}{\partial y}-2 \frac{W e}{W e_{R}} \hat{T}_{y y}\left(\frac{\sigma+3 \beta^{\star}}{\sigma}-\frac{\sqrt{3}\left(1-\beta^{\star}\right)}{\sqrt{\sigma}}\right) \\
& -\frac{T_{B y y} W e^{2} \operatorname{tr} \hat{\mathbf{T}}}{\sigma W e_{R}(1-\beta)}\left(\frac{\sqrt{3}\left(1-\beta^{\star}\right)}{\sqrt{\sigma}}+\frac{6 \beta^{\star}}{\sigma}\right)-\frac{W e \sqrt{3 / \sigma} t r \hat{\mathbf{T}}}{W e_{R} \sigma}-\hat{T}_{y y}, \\
& W e\left[\omega \hat{T}_{z z}+u_{B x} \frac{\partial \hat{T}_{z z}}{\partial x}+u_{B y} \frac{\partial \hat{T}_{z z}}{\partial y}+\hat{u}_{x} \frac{\partial T_{B z z}}{\partial x}+\hat{u}_{y} \frac{\partial T_{B z z}}{\partial y}\right]-2 k W e \hat{u}_{z} T_{B z z} \\
& =2(1-\beta) k \hat{u}_{z}-2 \frac{W e}{W e_{R}} \hat{T}_{z z}\left(\frac{\sigma+3 \beta^{\star}}{\sigma}-\frac{\sqrt{3}\left(1-\beta^{\star}\right)}{\sqrt{\sigma}}\right) \\
& -\frac{T_{B z z} W e^{2} \operatorname{tr} \hat{\mathbf{T}}}{\sigma W e_{R}(1-\beta)}\left(\frac{\sqrt{3}\left(1-\beta^{\star}\right)}{\sqrt{\sigma}}+\frac{6 \beta^{\star}}{\sigma}\right)-\frac{W e \sqrt{3 / \sigma} t r \hat{\mathbf{T}}}{W e_{R} \sigma}-\hat{T}_{x x},
\end{aligned}
$$




$$
\begin{aligned}
& W e\left[\omega \hat{T}_{x y}+u_{B x} \frac{\partial \hat{T}_{x y}}{\partial x}+u_{B y} \frac{\partial \hat{T}_{x y}}{\partial y}+\hat{u}_{x} \frac{\partial T_{B x y}}{\partial x}+\hat{u}_{y} \frac{\partial T_{B x y}}{\partial y}\right]- \\
& W e\left[\frac{\partial \hat{u}_{x}}{\partial x} T_{B x y}+\frac{\partial \hat{u}_{x}}{\partial y} T_{B y y}+\frac{\partial u_{B x}}{\partial x} \hat{T}_{x y}+\frac{\partial u_{B x}}{\partial y} \hat{T}_{y y}\right] \\
& -W e\left[\frac{\partial \hat{u}_{y}}{\partial x} T_{B x x}+\frac{\partial \hat{u}_{y}}{\partial y} T_{B x y}+\frac{\partial u_{B y}}{\partial x} \hat{T}_{x x}+\frac{\partial u_{B y}}{\partial y} \hat{T}_{x y}\right] \\
& =(1-\beta)\left(\frac{\partial \hat{u}_{x}}{\partial y}+\frac{\partial \hat{u}_{y}}{\partial x}\right)-2 \frac{W e}{W e_{R}} \hat{T}_{x y}\left(\frac{\sigma+3 \beta^{\star}}{\sigma}-\frac{\sqrt{3}\left(1-\beta^{\star}\right)}{\sqrt{\sigma}}\right) \\
& -\frac{T_{B x y} W e^{2} \operatorname{tr} \hat{\mathbf{T}}}{\sigma W e_{R}(1-\beta)}\left(\frac{\sqrt{3}\left(1-\beta^{\star}\right)}{\sqrt{\sigma}}+\frac{6 \beta^{\star}}{\sigma}\right)-\frac{W e \sqrt{3 / \sigma} t r \hat{\mathbf{T}}}{W e_{R} \sigma}-\hat{T}_{x y}, \\
& W e\left[\omega \hat{T}_{x z}+u_{B x} \frac{\partial \hat{T}_{x z}}{\partial x}+u_{B y} \frac{\partial \hat{T}_{x z}}{\partial y}\right]-W e\left[\frac{\partial \hat{u}_{z}}{\partial x} T_{B x x}+\frac{\partial \hat{u}_{z}}{\partial y} T_{B x y}-k \hat{u}_{x} T_{z z}\right] \\
& -W e\left[\frac{\partial u_{x}}{\partial x} \hat{T}_{x z}+\frac{\partial u_{x}}{\partial y} \hat{T}_{y z}\right]=(1-\beta)\left(-k \hat{u}_{x}+\frac{\partial \hat{u}_{z}}{\partial x}\right) \\
& -2 \frac{W e}{W e_{R}} \hat{T}_{x z}\left(\frac{\sigma+3 \beta^{\star}}{\sigma}-\frac{\sqrt{3}\left(1-\beta^{\star}\right)}{\sqrt{\sigma}}\right)-\hat{T}_{x z}, \\
& W e\left[\omega \hat{T}_{y z}+u_{B x} \frac{\partial \hat{T}_{y z}}{\partial x}+u_{B y} \frac{\partial \hat{T}_{y z}}{\partial y}\right]-W e\left[\frac{\partial \hat{u}_{z}}{\partial x} T_{B x y}+\frac{\partial \hat{u}_{z}}{\partial y} T_{B y y}-k \hat{u}_{y} T_{z z}\right] \\
& -W e\left[\frac{\partial u_{y}}{\partial x} \hat{T}_{x z}+\frac{\partial u_{y}}{\partial y} \hat{T}_{y z}\right] \\
& =(1-\beta)\left(-k \hat{u}_{y}+\frac{\partial \hat{u}_{z}}{\partial y}\right) \\
& -2 \frac{W e}{W e_{R}} \hat{T}_{y z}\left(\frac{\sigma+3 \beta^{\star}}{\sigma}-\frac{\sqrt{3}\left(1-\beta^{\star}\right)}{\sqrt{\sigma}}\right)-\hat{T}_{y z} .
\end{aligned}
$$

The existence of a non-trivial solution to the resulting equations is an eigenvalue problem for $\omega$, which defines the stability spectrum. If the leading (complex) eigenvalue has negative real part then the disturbances decay, the variables return to their base state and the flow is said to be stable. If, on the otherhand, the real part of $\omega$ is greater than zero the disturbances grow in time and the flow is unstable. We are interested in examining purely elastic flow instabilities, that is those occurring in the absence of inertial forces. Thus all predictions which follow were obtained with $R e=0$.

\subsection{Numerical Method}

The semi-staggered dilation-free finite volume method of Sahin $[30,31,37]$ is adopted for the Rolie-Poly constitutive equation for the extra stress and applied to the constriction flows discussed in Sections 1 and 3. This method offers the 
advantage of better pressure coupling than a collocated approach while remaining geometrically flexible and relatively simple. Moreover, the discretisation of the continuity equation is reduced to the domain boundary, which is important for global mass conservation. Further details about the numerical method can be found in Sahin [31, 37].

The governing equations (4), (5) and (6) are discretised in time using the (first-order) implicit Euler method and in space using a semi-staggered finite volume mesh [31, 37]; an example of the (quadrilateral) element used to construct the computational domain is shown in Figure 5. The time-dependent finite volume discretisation leads to a linear system where the extra stresses are coupled with the velocity and pressure field. A time-splitting technique with a combination of upper-triangular and Krylov subspace preconditioners are used to decouple and efficiently solve the system [31, 37, 38]. The continuity (4) and constitutive (6) equations are integrated over a quadrilateral element $\Omega_{e}$ and the momentum equation is integrated over an arbitrary irregular dual control volume $\Omega_{d}$. In this formulation the pressure and stress are defined at element centroids while the velocity is defined at the nodal points.

Once the steady base flow has been determined, we proceed to the analysis of its stability using equations (9)-(18). The discrete system for the perturbed equations take the form of a generalised eigenvalue problem which is solved using the shift-invert Arnoldi method [30, 33]. The MUMPS library [32], which is a direct method based on LU decomposition for the solution of sparse systems, is utilised to solve all the discrete algebraic equations.

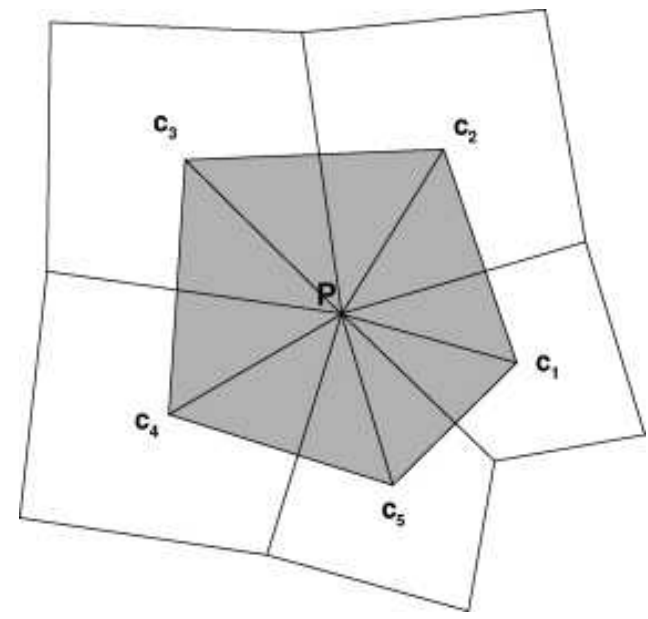

Figure 5: Two-dimensional, unstructured, mesh with a dual control volume surrounding a node $\mathrm{P}$. The centroids of the elements are denoted by $c_{i}$. 


\section{Numerical Experiments}

In this section we present the critical conditions for the instability of a Rolie-Poly fluid in two different forms of constriction flows that are relevant to the polymer-processing industry: the bumpy wall geometry, which is a sheardominated flow; and the 8:1:8 contraction-expansion geometry, which is extensiondominated. To assess the mechanisms responsible, we also compute the most unstable wavenumber, $k_{c r i t}$, and the Pakdel and McKinley non-dimensional grouping, $M$, [19] given by equation (1) using the reptation relaxation time, $\tau$. In a recent article [25], an experimental and numerical study of the stability of a polystyrene in 8:1:8 contraction-expansion flow was documented. For consistency and comparison, unless otherwise stated we take $\beta^{\star}=0.283,3 Z=20$ and $\beta=0.1$.

\subsection{Bumpy Wall}

Our first geometry is a channel with a periodic array of half cylinders placed on the horizontal wall. The cylinder spacing is $L=2.5 R$ and the height of the channel is $H=4 R$, where $R=1$ is the radius of the cylinder. The dimensionless numbers that characterise the flow are the Weissenberg numbers based on the reptation and Rouse relaxation times, $W e=\tau\langle u\rangle / R$ and $W e_{R}=\tau_{R}\langle u\rangle / R$, respectively, where $\langle u\rangle$ is the average velocity. The viscosity ratio is $\beta=\eta_{s} /\left(\eta_{p}+\right.$ $\eta_{s}$ ), where $\eta_{s}$ and $\eta_{p}$ are the solvent and polymer viscosities. In the stability calculation, the wavenumber is nondimensionilized with the cylinder radius, $R$. To check the dependency of the results on the discrete domain a series of mesh refinements were conducted by multiplying the mesh sizes by $1 / \sqrt{2}$ in each direction. All results shown in this section were obtained using 36201 nodes and 35644 elements.

Table 1 shows the dependence of the critical Weissenberg number on the solvent viscosity $\beta$ and gives the most unstable wavenumber, $k_{\text {crit }}$. Clearly, viscosity acts to damp oscillations and the flow has weaker stability properties for smaller $\beta$. Figure 6 plots the computed $U$ - and $V$-velocity contours, with streamlines, of the typical base flow of a Rolie-Poly fluid in the bumpy wall geometry at the critical Weissenberg number of $W e_{R_{c r i t}}=22.47$. The $T_{x x}$ component of the stress is shown in Figure 7. We notice a separation region between the cylinders, as expected, with large stress coming off the cylinder walls.

The dependency of the numerical solutions on the grid size has been investigated with a series of mesh refinements. Table 2 shows the critical Weissenberg number on four successive meshes. The parameters for this study were $3 Z=20$, $\beta^{\star}=0.283$ and $\beta=0.1$.

More revealing is the role of the underlying polymeric structure on the flow. Table 3 shows the influence of the CCR parameter $\beta^{\star}$ on the stability of the material which has an entanglement number of $Z=20 / 3$ (or $\tau / \tau_{R}=W e / W e_{R}=$ 20 ) and also gives the most unstable wavenumber, $k_{\text {crit }}$ along with the critical Pakdel and McKinley number, $M_{\text {crit }}=\max \{M(x, y)\}$. The data suggests that convective constraint release does indeed affect the flow but it is considerably 


\begin{tabular}{|c|c|c|}
\hline$\beta$ & $W e_{R_{\text {crit }}}$ & $k_{\text {crit }}$ \\
\hline 0.05 & 11.87 & 12 \\
0.1 & 22.47 & 14 \\
0.2 & 44.98 & 14 \\
0.3 & 57.34 & 16 \\
0.5 & $/$ & $/$ \\
\hline
\end{tabular}

Table 1: Influence of the viscosity $\beta$ on stability in a bumpy wall geometry using a Rolie-Poly model with $\beta^{\star}=0.283$ and $3 Z=20$. The '/ denotes no instability found.

\begin{tabular}{|c|c|c|}
\hline Number of nodes & $W e_{R_{\text {crit }}}$ & $k_{\text {crit }}$ \\
\hline 10210 & 22.02 & 16 \\
19027 & 22.36 & 14 \\
36201 & 22.47 & 14 \\
72587 & 22.53 & 14 \\
\hline
\end{tabular}

Table 2: Convergence of the critical Weissenberg number for flow in the bumpy wall geometry when $3 Z=20, \beta^{\star}=0.283$ and $\beta=0.1$.

weaker than that of the solvent viscosity. For the bumpy wall geometry with a molecular weight determined by $3 Z=20$ it appears that convective constraint release, which is a stretch-dependent relaxation, has a stabilising effect. That is, increasing the amount of convective-constraint release permits modestly larger flow rates. The most unstable wavenumber is reasonably large and the critical Pakdel and McKinley number is close to unity, suggesting the curvature of streamlines instability mechanism. Moreover, Figures 8 and 9 indicates that when $3 Z=20, M$ is largest along the streamline coming off the bumps where the instability originates, indicating again that we are seeing the classic curved streamline mechanism.

The molecular weight of a fluid is of significant interest and importance when addressing the stability of a polymeric material and in our non-dimensional formulation of the Rolie-Poly fluid this quantity is expressed by the entanglement number, $Z$, or the ratio of the Rouse and reptation Weissenberg numbers. Ta-

\begin{tabular}{|c|c|c|c|}
\hline$\beta^{\star}$ & $W e_{R_{\text {crit }}}$ & $k_{\text {crit }}$ & $M_{\text {crit }}$ \\
\hline 0.1 & 22.02 & 14 & 0.96 \\
0.3 & 22.46 & 13 & 0.94 \\
0.5 & 22.57 & 12 & 0.97 \\
0.7 & 23.70 & 12 & 0.98 \\
0.9 & 23.93 & 12 & 0.98 \\
\hline
\end{tabular}

Table 3: Influence of the CCR parameter $\beta^{\star}$ on stability in a bumpy wall geometry using a Rolie-Poly model with $\beta=0.1$ and $3 Z=20$. 

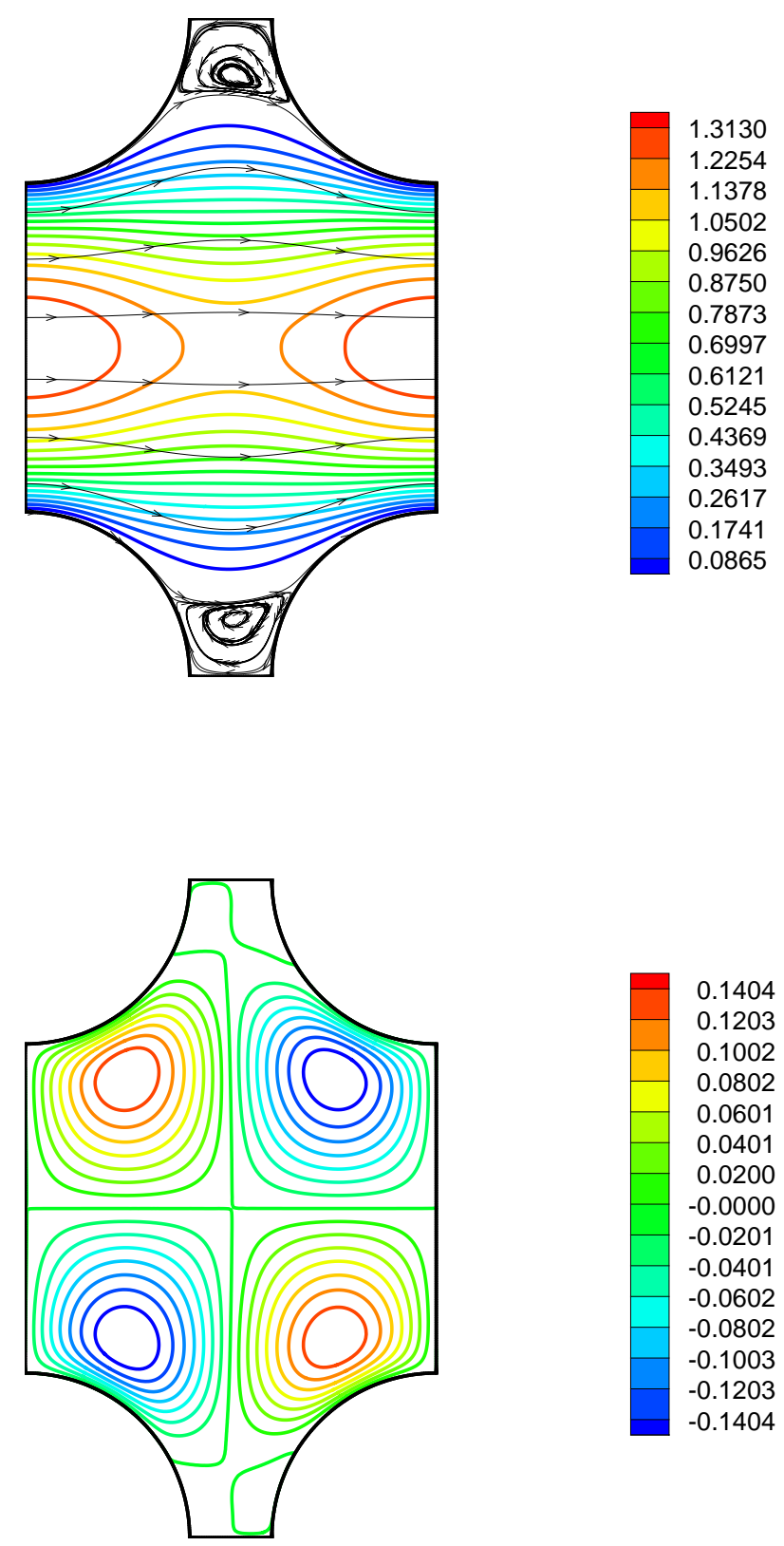

Figure 6: The computed contours and streamlines for the steamwise $\left(u_{x}\right.$, top) and spanwise $\left(u_{y}\right)$ components of the velocity for a Rolie-Poly fluid in a bumpy wall flow at the critical Weissenberg number of $W e_{R_{\text {crit }}}=22.47$. The parameters for the flow are: $\beta^{\star}=0.283$, $\beta=0.1$ and $3 Z=20$. 


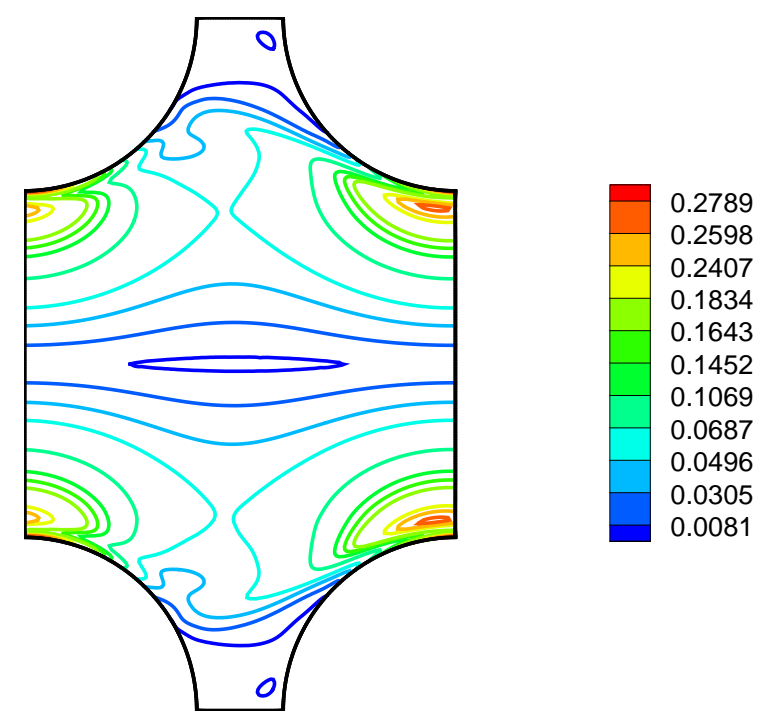

Figure 7: The computed contours for the $T_{x x}$ component of the stress of a Rolie-Poly fluid in a bumpy wall flow at the critical Weissenberg number of $W e_{R_{\text {crit }}}=22.47$. The parameters for the flow are: $\beta^{\star}=0.283, \beta=0.1$ and $3 Z=20$. 


\begin{tabular}{|c|c|c|c|}
\hline $3 Z$ & $W e_{R_{\text {crit }}}$ & $k_{\text {crit }}$ & $M_{\text {crit }}$ \\
\hline 20 & 22.47 & 13 & 0.94 \\
30 & 21.74 & 10 & 0.93 \\
40 & 21.02 & 9 & 0.94 \\
\hline
\end{tabular}

Table 4: Influence of the number of entanglements on stability in a bumpy wall geometry using a Rolie-Poly model with $\beta^{\star}=0.283$ and $\beta=0.1$.

ble 4 contains $W e_{R_{c r i t}}$ as a function of $Z$ and clarifies its influence. The flow is more stable when the the number of entanglements of the polymeric material decreases. This suggests that materials with this architecture are able to maintain stability at greater flow rates in such a shear-dominated flow. The flows are qualitatively similar to those above, so the curved-streamline instability is once again suspected. We did not find any instabilities in the bumpy wall flow when $1<3 Z<20$ on the tested meshes. However, we were able to predict instabilities when the entanglement number is very small $(3 Z \ll 1)$. Although the fluid could still be subjected to shear-banding at sufficiently large Weissenberg numbers, the instability manifests itself at much smaller shear rates, before the constitutive curve loses monotonicity. As an example, we found a critical Weisenberg number of $W e_{\text {crit }}=2.3$ and a critical wavenumber of $k_{\text {crit }}=2$ when $\beta^{\star}=0.283, \beta=0.67$ and $3 Z=0.01$. This is of the same order as the critical Weissenburg number reported in [30] for an Oldroyd-B fluid. This is no coincidence: we can see from equation (6) that the Rolie-Poly equation appears to be similar to the Oldroyd-B model when $W e / W e_{R} \rightarrow 0$. We must stress, however, that this is not the "Oldroyd-B limit" of the Rolie-Poly constitutive equation; this is instead $2(1-\sqrt{3 / \operatorname{tr} \mathbf{T}}) / \tau_{R} \rightarrow 0$. The limit $\tau_{R} \rightarrow \infty$ is thus not sufficient; one also requires $\tau_{R} \gg 1 / \sqrt{\operatorname{tr} \mathbf{T}}$, which is not guaranteed for small extra stresses.

\subsection{8:1:8 Contraction-Expansion}

To further our understanding of the cause of instability in linear polymers. we simulate the benchmark 8:1:8 contraction-expansion flow using a RoliePoly model and again probe the parameter space. The dimensionless numbers that characterise the flow are the Weissenberg numbers based on the reptation and Rouse relaxation times, $W e=\tau\langle u\rangle / R$ and $W e_{R}=\tau\langle u\rangle / R$, respectively, where $\langle u\rangle$ is the average velocity and $R=1$ is the radius of the contraction. In the stability calculation, the wavenumber is nondimensionilized with the contraction radius, $R$. Since we are concerned with the influence of the polymer physics on the stability of flow we only present results for variations in the CCR parameter and the molecular weight (number of entanglements, $Z$ ) but note that the effect of solvent viscosity in this flow is similar to that in the bumpy wall case. To help understand the mechanism responsible, we also show the computed Pakdel and Mckinley number (1) for all flows that become unstable. The values of $M$ were found to be an order of magnitude lower than what is expected for curvature-driven instability (Tables 5, 6, 7), and we thus conclude 

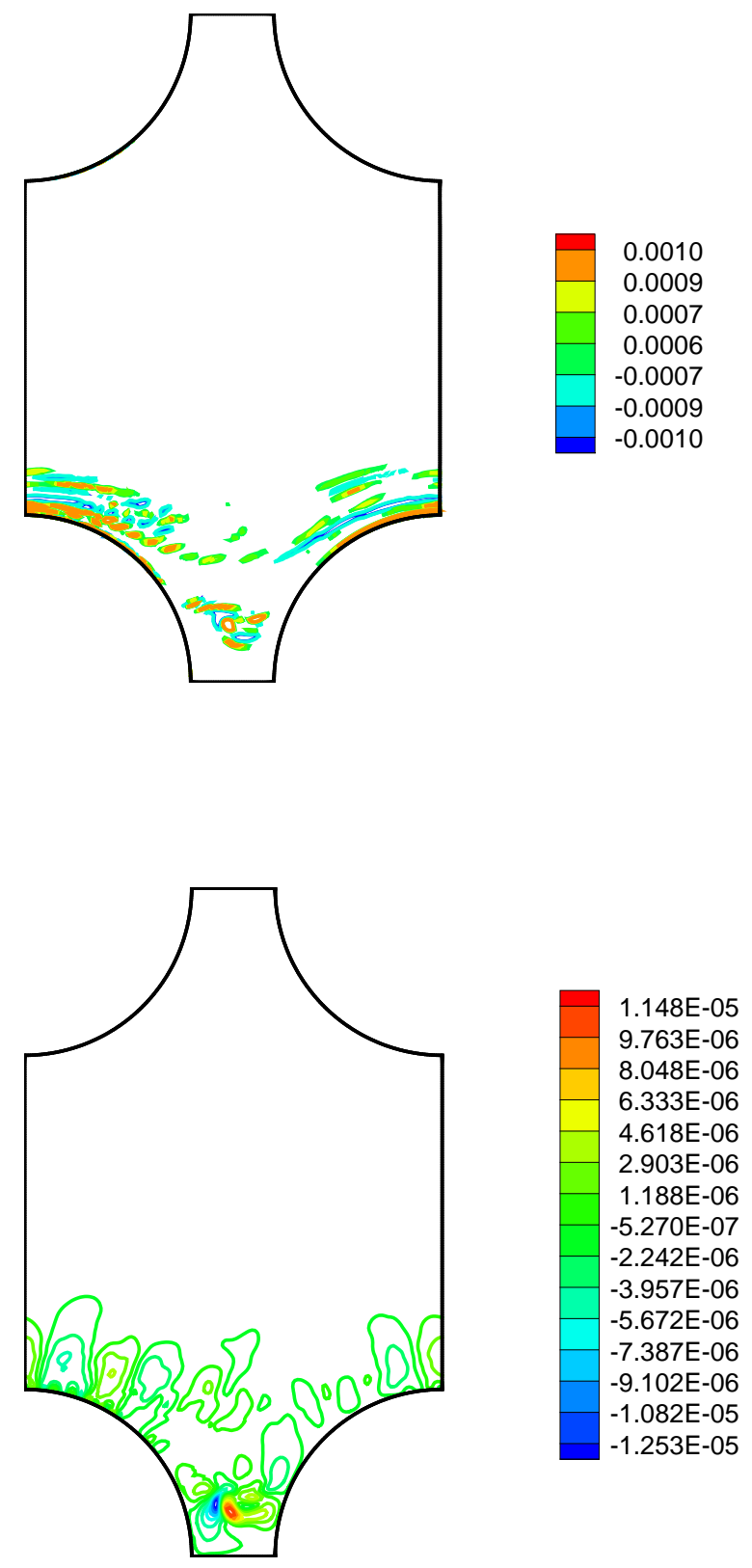

Figure 8: The computed contours of the $\hat{T}_{x x}$ component of stress (top) and $\hat{u}_{z}$ for a RoliePoly fluid in a bumpy wall flow at the critical Weissenberg number of $W e_{R_{\text {crit }}}=23.93$. The parameters for the flow are $\beta^{\star}=0.9, \beta=0.1$ and $3 Z=20$. 


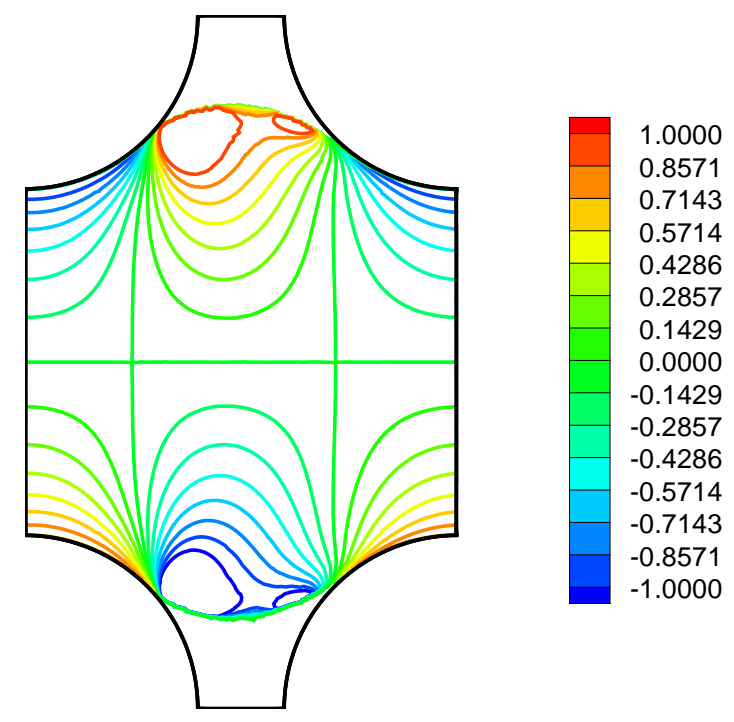

Figure 9: The computed contours of $M$ for a Rolie-Poly fluid in a bumpy wall flow at the critical Weissenberg number of $W e_{R_{\text {crit }}}=23.93$. The parameters for the flow are $\beta^{\star}=0.9$, $\beta=0.1$ and $3 Z=20$. 


\begin{tabular}{|c|c|c|c|}
\hline$\beta^{\star}$ & $W e_{R_{\text {crit }}}$ & $k_{\text {crit }}$ & $M_{\text {crit }}$ \\
\hline 0.1 & 7.23 & 3.5 & 0.202 \\
0.3 & 7.60 & 0.4 & 0.220 \\
0.5 & 8.25 & 0.8 & 0.257 \\
0.7 & 8.96 & 0.3 & 0.260 \\
0.9 & 9.14 & 0.3 & 0.261 \\
\hline
\end{tabular}

Table 5: Influence of the CCR parameter $\beta^{\star}$ on stability in a 8:1:8 contraction-expansion geometry using a Rolie-Poly model with $\beta=0.1$ and $3 Z=5$.

\begin{tabular}{|c|c|c|c|}
\hline$\beta^{\star}$ & $W e_{R_{\text {crit }}}$ & $k_{\text {crit }}$ & $M_{\text {crit }}$ \\
\hline 0.1 & 5.97 & 1.1 & 0.049 \\
0.3 & 6.89 & 0.2 & 0.053 \\
0.5 & 7.57 & 0.2 & 0.057 \\
0.7 & 7.80 & 0.1 & 0.058 \\
0.9 & 7.87 & 0.2 & 0.061 \\
\hline
\end{tabular}

Table 6: Influence of the CCR parameter $\beta^{\star}$ on stability in a 8:1:8 contraction-expansion geometry using a Rolie-Poly model with $\beta=0.1$ and $3 Z=20$.

that streamline curvature is not the dominant cause here. All results shown in this section were obtained using 47614 elements and 45968 node.

Tables 5 and 6 show the influence of the CCR parameter $\beta^{\star}$ on the stability of the material when the entanglement number is given by $3 Z=5$ and $3 Z=20$, respectively. The results show that convective constraint release does indeed effect the stability of flow in the 8:1:8 contraction-expansion geometry. We reiterate our belief that this is due to polymer chain stretch and argue that the relationship between convective constraint release and chain stretch is governed by the dominating flow dynamics; our reasoning will be discussed further in Section 4. The computed contours of $\hat{T}_{x x}$ and $\hat{u}_{z}$ when $\beta^{\star}=0.5$ and $3 Z=20$ are plotted in Figure 10. We notice a fluctuation along the centerline upstream of the contraction and two perturbations on the inside wall downstream of the contraction, which is the source of the instability. The upstream activity is affected by the downstream instability.

The influence of the entanglement number on the stability of this 8:1:8 contraction-expansion flow is given in Table 7. We see that materials with a smaller entanglement number, and therefore less stretch, are able to maintain stability at greater flow rates (we note again that we discuss stability in terms of the smallest Weissenberg number, $W e_{R}$ ). The computed contours of $\hat{T}_{x x}$ and $\hat{u}_{z}$ when $3 Z=5$ are plotted in Figure 11 . We notice a qualitative difference compared to the plots of Figure 10. In particular, we now see two asymmetric negative streaks running through the contraction and a single, stronger, perturbation off the inside wall downstream of the contraction. This suggests that chain stretch may not only influence the critical Wessenberg number but also 


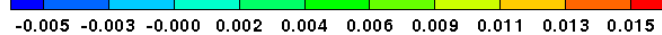

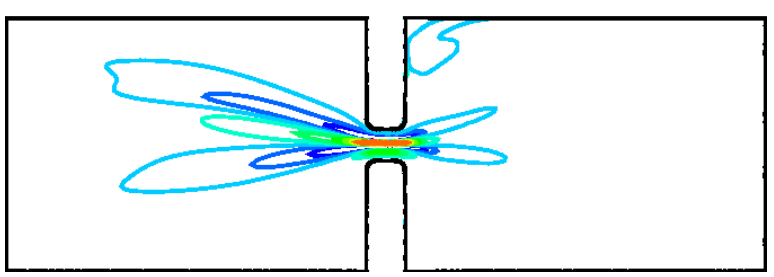

$\begin{array}{llllllllll}-0.0008 & -0.0007 & -0.0006 & -0.0005 & -0.0004 & -0.0003 & -0.0002 & -0.0001 & 0.0000 & 0.0001\end{array}$

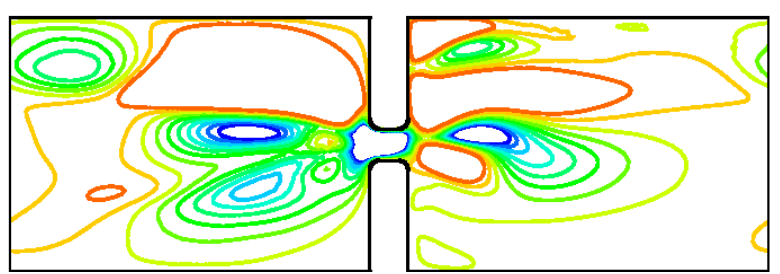

Figure 10: The computed contours for $\hat{T}_{x x}$ component of stress (top) and $\hat{u}_{z}$ of a Rolie-Poly fluid in a 8:1:8 contraction-expansion flow at the critical Weissenberg number of $W e_{R_{\text {crit }}}=$ 7.57. The parameters for the flow are: $\beta^{\star}=0.5, \beta=0.1$ and $3 Z=20$. 


\begin{tabular}{|c|c|c|c|}
\hline $3 Z$ & $W e_{R_{\text {crit }}}$ & $k_{\text {crit }}$ & $M$ \\
\hline 2 & 10.20 & 0.7 & 0.527 \\
5 & 8.01 & 0.4 & 0.200 \\
10 & 7.13 & 0.3 & 0.091 \\
20 & 6.94 & 0.2 & 0.051 \\
40 & 6.90 & 0.1 & 0.038 \\
\hline
\end{tabular}

Table 7: Influence of the entanglement number on the stability in a 8:1:8 contraction-expansion geometry using a Rolie-Poly model with $\beta^{\star}=0.283$ and $\beta=0.1$.

effects the nature of instability in polymeric materials. Note also the smallness of the critical wavenumber $k_{c r i t}$ compared with the bumpy wall experiments.

\section{Discussion}

The connection between a polymeric fluid's molecular dynamics and its stability properties has been investigated using the Rolie-Poly constitutive model for two varieties of contraction-expansion flow - one shear dominated, the other extension dominated. At least two distinct instabilities which can be understood with reference to the underlying polymer physics were found.

The instabilities in the 8:1:8 contraction-expansion flow geometry are essentially in-plane (small out-of-plane wavenumber, $k R / 2 \pi$ ) and are triggered in the extension-dominated regions. This claim is further supported by the calculation of the Pakdel and McKinley number of equation (1), which naturally leads to the conclusion that streamline curvature is not (solely) responsible for the observed instabilities. We interpret our data in terms of the the smallest of the two Weissenberg numbers, that is the Rouse Weissengerg number, $W e_{R}$. Table 7 shows that a material with a reduced number of entanglements (equivalently, decreasing $W e / W e_{R}$ in the non-dimensional formulation) inhibits instability (in terms of $W e_{R}$ ), in agreement with the work of Hassell et al. [25] and Su and Khomami [24]. This corresponds to a low molecular weight fluid with a smaller entanglement number. Although polymeric materials with a lower molecular weight are associated with a smaller entanglement number (and thus less chain stretch), all the Rolie-Poly fluids studied here must be considered to be highly elastic. Note that even when $3 Z=5$, the critical Weissenberg number $W e_{R_{\text {crit }}} \sim O(10)$, as shown in Table 5 . However, we can infer from the figures in Section 2.1 that at high shear rates the shear viscosity decreases. The flow progressively disentangles the polymers, causing the fluid to shear-thin. This results in a reduction of the base flow stresses and thus a more stable flow. Conversely, fluids with a higher molecular weight are yet more entangled, so experience less shear thinning at the same shear rate, and therefore have a higher effective Weissenberg number. The base flow stresses are thus larger and hence the greater the prospect of elastic instability.

A discussion of the shear behavior of Rolie-Poly fluids in the contractionexpansion geometry is necessary but not sufficient to understand its stability 
\begin{tabular}{ll|lll|l|l|l|l|l|l}
\hline & & & & & & & & & \\
-0.005 & -0.003 & -0.001 & 0.002 & 0.004 & 0.006 & 0.008 & 0.011 & 0.013 & 0.015
\end{tabular}

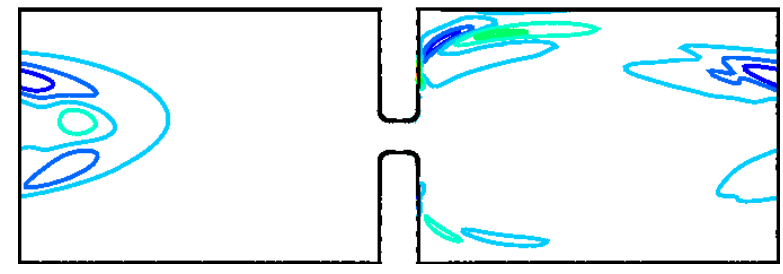

$\begin{array}{lllllllllll} & -0.0008 & -0.0007 & -0.0006 & -0.0005 & -0.0004 & -0.0003 & -0.0002 & -0.0001 & 0.0000 & 0.0001\end{array}$

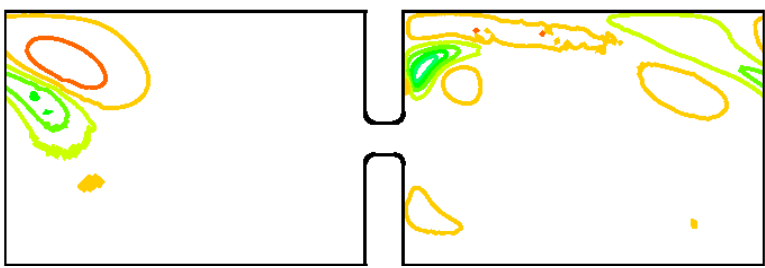

Figure 11: The computed contours for $\hat{T}_{x x}$ component of stress (top) and $\hat{u}_{z}$ of a Rolie-Poly fluid in a 8:1:8 contraction-expansion flow at a the critical Weissenberg number of $W e_{R_{\text {crit }}}=$ 8.25. The parameters for the flow are: $\beta^{\star}=0.5, \beta=0.1$ and $3 Z=5$. 
properties in terms of the molecular structure since the flow is dominated by its extensional components. Referring to the rheological response of the RoliePoly model in planar extensional flow, Figures 3 and 4 show that increasing the strain-rate increases the the extensional viscosity, which in turn produces large tensile stresses that break the flow symmetry, thus causing instabilities. We can also infer from the plots that there is a smaller region of negative gradient in the extensional viscosity for $3 Z=5$ than for $3 Z=20$. Together with the numerical simulations, this leads to the colclusion that any strain softening experienced by the fluid will be detrimental to its stability. Tables 5 and 6 show that the CCR parameter has a stabilising effect in the 8:1:8 contraction-expansion flow. This can be interpreted as follows: In an extension-dominated flow, as more constraints are released (corresponding to a larger CCR parameter) the diameter of the constraining tube increases and thus the polymer experiences less tube "squeezing". Therefore, the amount of chain stretch is reduced and the stability of the flow in enhanced.

The form of the instability in the shear-dominated bumpy wall flow, on the other-hand, leads us to believe that the curvature of the streamlines (elastic Taylor rolls) does indeed trigger instability. Here the Pakdel and Mckinley number is of unit order and the critical wavenumber, $k R / 2 \pi$, is comparable to the characteristic lengthscale of the flow. Table 4 shows that, when $\beta^{\star}=0.283$, polymer melts with more entanglements are more stable. We again attribute this to the shear-thinning behavior, as discussed above. By comparing Table 4 and Table 7 we notice that $W e_{R_{\text {crit }}}$ drops by over $6 \%$ when we increase $3 Z$ from 20 to 40 in the bumpy wall geometry but by less than $0.6 \%$ in the contractionexpansion flow. This is becuase the bumpy wall is shear-dominated, as opposed to extension-dominated, causing greater disentanglement of polymers, in turn more shear-thinning, and thus a greater dependence of the critical Weissenburg number on $Z$.

The dependence of the critical Weissenberg number on the CCR parameter, as shown by Table 3, is of interest and gives us further physical insight. We have found that CCR has a stabilising effect. This property may be understood with the aid of Figure 2 which shows that increasing CCR in steady shear flow ensures a positive gradient of the constitutive curve (monotonicity), a smaller plateau region, and hence more stable behaviour. The calculations suggest that the effect of CCR on the stability of the flow is rather weak. This is also in agreement with Figure 2 which shows that that convective constraint release has only a slight influence on the first normal stress different in steady shear flow. The flow of Rolie-Poly fluid in constrictions with $3 Z \gg 1$ is far more stable than that of an Oldroyd-B fluid. The Oldroyd-B fluid predicts a zero second normal stress difference, while Figures 3 and 4 show that the Roly-Poly model has $N_{2}>0$. Moreover, we see that $N_{2}$ is greater when $3 Z=5$ than when $3 Z=20$. This supports the observations of previous research [9] which states that an appreciable second normal stress difference has a stabilising effect.

The numerical experiments confirm that chain stretch and the degree of entanglement have an influence on the stability of entangled polymeric materials. We remark that Somasi and Khomami [10] found, via stochastic simulations of 
Hookean and FENE dumbbells, that chain stretch (that is, the extensibility of the dumbbells) has a destabilising effect in Couette flow of unentangled polymer solutions; also reasoning with reference to the relationship between molecular properties and shear-thinning behaviour. Although the physics of dilute polymer solutions is different to entangled polymer melts, it is interesting to note this common characteristic. As a direction for further research we suggest that an investigation of the effect of the geometry on the flow stability would be insightful and could increase our understanding of the relationship between elastic instabilities and the molecular structure of the material. In particular, we speculate that the role of convective-constraint release in the shear dominated bumpy wall flow is likely to be different for different distances between the cylinders and different entanglement number. Consider a polymer chain confined to a "tube" formed by the surrounding polymers. Shear-dominated flows cause such chains to become highly stretched and aligned with the flow. Therefore, the dynamics of the polymer is altered very little by the surrounding flow. However, if some of the tube constraints are released then segments of the polymer could, in principle, escape from the tube and fall out of line with the flow. It will then be caught by the external shearing motion and thus stretched even further. In other words, in a highly shear-dominated flow (one which does not trigger an appreciable extensional flow) greater convective constraint release could increases chain stretch and, as a result, induce instability. Indeed, the spacing between the half-cylinders will influence the amount of shear and stretch the fluid experiences. This agrees with the conclusions drawn by previous authors when studying Taylor-Couette flow [19, 20]: when polymers are displaced from a streamline the chain becomes stretched by the shearing motion of the flow, which in turn amplifies the non-Newtonian hoop stress and the perturbation.

We note briefly that the single-mode Rolie-Poly model shows a disagreement with the full linear theory [39] for large shear rates due to the assumption of uniform stretch, as discussed in [2]. Although one could use a multi-mode RoliePoly equation (at a greater computational cost), it would still not be the most general model. It is, however, the most sophisticated formulation of the DoiEdwards tube model that is also computationally viable. Broadly speaking, the use of this molecular constitutive model together with a sophisticated numerical algorithm has enabled us to understand the physics of elastic instabilities in viscoelastic materials in terms of their underlying polymer dynamics.

\section{Acknowledgements}

We would like to think Dr Mehmet Sahin and Dr Ian Griffiths for their helpful advice and discussions. We would also like to thank the anonymous referees for their pertinent comments which helped improve the quality of this article. This work was funded through the Microscale Polymer Processing project $\left(\mu P P^{2}\right)$, EPSRC GR/T11807/01. This publication was based on work supported in part by Award No KUK-C1-013-04, made by King Abdullah University of Science and Technology (KAUST). 
[1] M. Doi, S. F. Edwards, The Theory of Polymer Dynamics, Clarendon Press, Oxford, 1986.

[2] A. E. Likhtman, R. S. Graham, Simple constitutive equation for linear polymer melts derived from molecular theory: Rolie-Poly equation., J. Non-Newtonian Fluid Mech. 114 (2003) 1-12.

[3] C. C. Lin, The Theory of Hydrodynamic stability, Cambridge Univ. Press, Cambridge, 1955.

[4] T. C. Ho, M. M. Denn, Stability of plane poiseuille flow of a highly elastic liquid., J. Non-Newtonian Fluid Mech. 3 (1977) 179-195.

[5] H. J. Wilson, M. Renardy, Y. Y. Renardy, Structure of the spectrum in zero Reynolds number shear flow of the UCM and Oldroyd-B liquids, J. Non-Newtonian Fluid Mech. 80 (1999) 251-268.

[6] A. S. Palmer, T. N. Phillips, Numerical approximation of the spectra of phan-thien-tanner liquids., Numerical Algorithms 37 (2003) 133-153.

[7] A. M. Grillet, A. C. B. Bogaerds, G. W. M. Peters, F. P. T. Baaijens, Stability analysis of constitutive equations for polymer melts in viscometric flows, J. Non-Newtonian Fluid Mech. 103 (2001) 221-250.

[8] M. A. A. Öztekin, R. A. Brown, G. H. McKinley, Quantitative prediction of the viscoelastic instability in cone-and-plate flow of a boger fluid using a multi-mode giesekus model, J. Non-Newtonian Fluid Mech. 54 (2002) $351-377$.

[9] A. C. B. Bogaerds, A. M. Grillet, G. W. M. Peters, F. P. T. Baaijens, Stability analysis of polymer shear flows using the extended pom-pom constitutive equations, J. Non-Newtonian Fluid Mech. 108 (2002) 187-208.

[10] M. Somasi, B. Khomami, Linear stability and dynamics of viscoelastic flows using time-dependent stochastic simulation techniques, J. Non-Newtonian Fluid Mech. 93 (2000) 339-362.

[11] M. G. H. M. Baltussen, M. A. Hulsen, G. W. M. Peters, Numerical simulation of the fountain flow instability in injection molding, J. Non-Newtonian Fluid Mech. 165 (2010) 631-640.

[12] A. C. B. Bogaerds, M. A. Hulsen, G. W. M. Peters, F. P. T. Baaijens, Stability analysis of injection molding flows, J. Rheol. 48 (2004) 765-785.

[13] H. J. Wilson, J. M. Rallison, Short wave instability of co-extruded elastic liquids with matched viscosities, J. Non-Newtonian Fluid Mech. 80 (1999) $251-268$.

[14] J. C. Miller, J. M. Rallison, Instability of coextruded elastic liquids at high weissenberg number, J. Non-Newtonian Fluid Mech. 143 (2007) 88-106. 
[15] J. Yerushalmi, S. Katz, R. Shinnar, The stability of steady shear flows of some viscoelastic fluids, Chem. Eng. Sci. 25 (1970) 1891-1902.

[16] S. M. Fielding, H. J. Wilson, Shear banding and interfacial instability in planar poiseuille flow, J. Non-Newtonian Fluid Mech. 165 (2010) 196-202.

[17] R. G. Larson, E. S. G. Shaqfeh, S. J. Muller, A purely elastic instability in Taylor-Couette flow, J. Fluid Mech. 218 (1990) 573-600.

[18] G. H. McKinley, J. A. Byars, R. A. Brown, R. C. Armstrong, Observations on the elastic instability in cone-and-plate and parallel-plate flows of a polyisobutylene boger fluid, J. Non-Newtonian Fluid Mech. 40 (1991) 201229.

[19] P. Pakdel, G. H. McKinley, Elastic instability and curved streamlines, Phys. Rev. Lett. 77 (1996) 2459-2462.

[20] R. G. Larson, S. J. Muller, E. S. G. Shaqfeh, The effect of fluid rheology on the elastic Taylor-Couette instability, J. Non-Newtonian Fluid Mech. 51 (1994) 195-225.

[21] M. A. Fardin, T. J. Ober, C. Gay, G. Gregoire, G. H. McKinley, S. Leroug, Criterion for purely elastic taylor-couette instability in the flows of shearbanding fuids, Europhys. Lett. 96 (2011) 44004.

[22] M. W. Collis, A. K. Lele, M. R. Mackley, R. S. Graham, D. J. Groves, A. E. Likhtman, T. M. Nicholson, O. G. Harlen, T. C. B. McLeish, L. R. Hutchings, C. M. Fernyhough, R. N. Young, Constriction flows of monodisperse linear entangled polymers: Multiscale modeling and flow visualization, J. Rheol. 49 (2005) 501-522.

[23] D. G. Hassell, J. Embery, T. C. B. McLeish, M. R. Mackley, An experimental evaluation of the formation of an instability in monodisperse and polydisperse polystyrenes, J. Non-Newtonian Fluid Mech. 157 (2009) 1-14.

[24] Y.-Y. Su, B. Khomami, Interfacial stability of multilayer viscoelastic fluids in slit and converging channel die geometries, J. Rheol. 36 (1992) 357-388.

[25] D. G. Hassell, M. R. Mackley, M. Sahin, H. J. Wilson, O. G. Harlen, T. C. B. McLeish, Molecular physics of a polymer engineering instability: Experiments and computation, Phys. Rev. E 77 (2008) 050801(R).

[26] M. D. Smith, Y. L. Joo, R. C. Armstrong, R. A. Brown, Linear stability analysis of flow of an Oldroyd-B fluid through a linear array of cylinders., J. Non-Newtonian Fluid Mech. 109 (2003) 13-50.

[27] A. W. Liu, Viscoelastic flow of polymer solutions around arrays of cylinders: comparison of experiment and theory, Ph.D. thesis, MIT, 1997. 
[28] M. A. Alves, F. T. Pinho, P. J. Oliveira, The flow of viscoelastic fluids past a cylinder: finite-volume high-resolution methods, J. Non-Newtonian Fluid Mech. 97 (2001) 207-232.

[29] R. J. Poole, M. A. Alves, F. T. Pinho, P. J. Oliveira, Plane sudden expansion flows of viscoelastic liquids, J. Non-Newtonian Fluid Mech. 146 (2007) 79-91.

[30] M. Sahin, H. J. Wilson, A parallel adaptive unstructured finite volume method for linear stability (normal mode) analysis of viscoelastic flows, J. Non-Newtonian Fluid Mech. 155 (2008) 1-14.

[31] M. Sahin, H. J. Wilson, A semi-staggered dilation-free finite volume method for the numerical solution of viscoelastic fluid flows on all-hexahedral elements, J. Non-Newtonian Fluid Mech. 147 (2007) 79-91.

[32] P. R. Amestoy, I. S. Duff, J. Y. L'Excellent, Multifrontal parallel distributed symmetric and unsymmetric solvers, Comput. Methods Appl. Mech.Eng. 184 (2000) 501-520.

[33] Y. Saad, Variations on Arnoldi's method for computing eigenelements of large non-symmetric matrices, Linear Algebra Appl. 34 (1980) 269-295.

[34] E. S. G. Shaqfeh, Purely elastic instabilities in viscometric flows, Ann. Rev. Fluid. Mech. 28 (1996) 129-185.

[35] J. V. Valerio, M. S. Carvalho, C. Tomei, Efficient computation of the spectrum of viscoelastic flows., J. Comp. Phys. 228 (2009) 1172-1187.

[36] R. G. Larson, T. Sridhar, L. G. Leal, G. H. McKinley, A. E. Likhtman, T. C. B. McLeish, Defnitions of entanglement spacing and time constants in the tube model, J. Rheol. 47 (2003) 809-818.

[37] M. Sahin, A preconditioned semi-staggered dilation-free finite volume method for the incompressible Navier-Stokes equations on all-hexahedral elements, Int. J. Numer. Methods Fluids 49 (2005) 959-974.

[38] A. E. Caola, Y. L. Joo, R. C. Armstrong, R. A. Brown, Highly parallel time integration of viscoelastic flows, J. Non-Newtonian Fluid Mech. 100 (2001) 191-216.

[39] A. E. Likhtman, T. C. B. McLeish, Quantitative theory for linear dynamics of linear entangled polymers, Macromolecules 35 (2002) 6332-6343. 